\title{
LE PRODUIT HARMONIQUE DES SUITES
}

\author{
par Bernard CANDELPERGHER et Marc-Antoine COPPO
}

ABSTRACT. By means of an involutary binomial transformation on complex sequences, we define a new product called "harmonic" because of its remarkable properties towards harmonic sums. The Euler series transformation allows one to deduce from these properties some new and remarkable identities.

\section{INTRODUCTION}

Dans l'espace $\mathbf{C}^{\mathbf{N}^{*}}$ des suites à valeurs complexes, on considère la transformation linéaire $D$ associant à toute suite $a=(a(1), a(2), a(3), \ldots)$ la suite $D(a)$ définie par

$$
D(a)(n+1)=\sum_{k=0}^{n}(-1)^{k}\left(\begin{array}{l}
n \\
k
\end{array}\right) a(k+1) \quad \text { pour tout } n \geq 0 .
$$

L'opérateur $D$ est un automorphisme involutif du $\mathbf{C}$-espace vectoriel $\mathbf{C}^{\mathbf{N}^{*}}$, c'est-à-dire

$$
a=D(D(a)) .
$$

Formellement, les suites $a$ et $D(a)$ sont liées par la relation d'Euler:

$$
\sum_{n \geq 1} D(a)(n) z^{n}=-\sum_{n \geq 1} a(n)\left(\frac{z}{z-1}\right)^{n}
$$


La relation précédente montre en particulier que la suite harmonique $n \mapsto \frac{1}{n}$ est invariante par $D$. En notant $\frac{1}{N}$ cette suite, on peut donc écrire

$$
D\left(\frac{1}{N}\right)=\frac{1}{N}
$$

Si $D(a) D(b)$ désigne le produit de Hadamard (i.e. le produit terme à terme) des suites $D(a)$ et $D(b)$, on définit un nouveau produit dans $\mathbf{C}^{\mathbf{N}^{*}}$, noté $\bowtie$, par la formule

$$
a \bowtie b=D(D(a) D(b)) .
$$

Il en résulte (par involutivité de $D$ ) que $D(a b)=D(a) \bowtie D(b)$. Muni du produit $\bowtie$, l'espace vectoriel $\mathbf{C}^{\mathbf{N}^{*}}$ est une $\mathbf{C}$-algèbre commutative, associative et unitaire (mais non-intègre), l'élément unité étant la suite $\delta_{0}=(1,0,0, \ldots)=D(\mathbf{1})$ où $\mathbf{1}$ est la suite $(1,1,1, \ldots)$. Une suite $a$ est inversible pour le produit $\bowtie$ si et seulement si $D(a)$ est inversible pour le produit de Hadamard (i.e. $D(a)(n) \neq 0$ pour tout $n)$.

Une expression explicite du produit $a \bowtie b$ est donnée par la formule suivante :

$$
(a \bowtie b)(n+1)=\sum_{0 \leq l \leq k \leq n}(-1)^{k-l}\left(\begin{array}{l}
n \\
k
\end{array}\right)\left(\begin{array}{l}
k \\
l
\end{array}\right) a(k+1) b(n+1-l) \quad(n \geq 0),
$$

qui permet de le calculer pour de petites valeurs de $n$; on obtient ainsi

$(a \bowtie b)(1)=a(1) b(1)$,

$(a \bowtie b)(2)=a(2) b(1)+a(1) b(2)-a(2) b(2)$,

$(a \bowtie b)(3)=a(3) b(1)+a(1) b(3)+2 a(2) b(2)-2 a(3) b(2)-2 a(2) b(3)+a(3) b(3)$

etc.

Le produit $\bowtie$ possède des propriétés remarquables vis-à-vis des sommes harmoniques qui justifient sa dénomination de produit harmonique. On démontre (Théorème 2) la relation suivante: pour toute suite $a$, on a l'identité

$$
\left(\frac{1}{N} \bowtie a\right)(n)=\frac{1}{n}(a(1)+a(2)+\cdots+a(n)) .
$$

De cette propriété d'harmonicité découlent plusieurs applications remarquables. On obtient notamment (Théorème 4) la formule suivante :

$$
\sum_{n \geq n_{1} \geq \cdots \geq n_{k} \geq 1} \frac{1}{n_{1} \ldots n_{k-1}} a\left(n_{k}\right)=\sum_{m=1}^{n}(-1)^{m-1}\left(\begin{array}{l}
n \\
m
\end{array}\right) \frac{1}{m^{k-1}} D(a)(m)
$$


qui s'applique à toute suite $a$ et pour tout entier $k \geq 1$. Dans le cas particulier où $a$ est la suite harmonique $\frac{1}{N}$, on retrouve la classique «formule de Dilcher» (cf. [2], [3], [5]):

$$
\sum_{n \geq n_{1} \geq \cdots \geq n_{k} \geq 1} \frac{1}{n_{1} \ldots n_{k}}=\sum_{m=1}^{n}(-1)^{m-1}\left(\begin{array}{l}
n \\
m
\end{array}\right) \frac{1}{m^{k}},
$$

dont on donne une formulation plus générale (Corollaire 8).

On introduit les nombres

$$
S^{(k)}(a)(n)=\sum_{n \geq n_{1} \geq \cdots \geq n_{k} \geq 1} \frac{1}{n_{1} \ldots n_{k-1}} a\left(n_{k}\right)
$$

qui apparaissent comme une généralisation naturelle des nombres harmoniques $c_{n}^{(k)}$ de Rota et Roman (cf. [9], [10]): on a en effet la relation $c_{n}^{(k)}=S^{(k)}\left(\frac{1}{N}\right)(n)$. Par transformation d'Euler, on obtient la relation

$$
\sum_{n \geq 1} \frac{D(a)(n)}{n^{k}} z^{n}=-\sum_{n \geq 1} \frac{1}{n} S^{(k)}(a)(n)\left(\frac{z}{z-1}\right)^{n}
$$

qui permet notamment, dans le cas où $a$ est la suite $n \mapsto \frac{1}{(2 n-1)^{2}}$, d'étendre une formule de Ramanujan ([1], chapitre 9, Entry 34) pour la constante de Catalan (Exemple $23 \mathrm{~d})$ ).

\section{PRÉLIMINAIRES: OPÉRATEURS DANS L'ESPACE DES SUITES}

\subsection{L'ISOMORPHISME $\Phi$}

Notation. Le $\mathbf{C}$-espace vectoriel $\mathbf{C}^{\mathbf{N}^{*}}$ des suites

$$
a=(a(1), a(2), a(3), \ldots, a(n), \ldots)
$$

à valeurs dans $\mathbf{C}$ est noté $\mathcal{E}^{*}$.

DÉFINITION 1. Si C $[[z]]$ désigne l'espace des séries formelles, on a un isomorphisme naturel :

$$
\Phi: \mathcal{E}^{*} \longrightarrow \mathbf{C}[[z]]
$$

défini par

$$
\Phi(a)(z)=\sum_{n \geq 0} a(n+1) \frac{z^{n}}{n !}=a(1)+a(2) z+a(3) \frac{z^{2}}{2}+a(4) \frac{z^{3}}{6}+\ldots .
$$


DÉFINITION 2. Les opérateurs sur $\mathcal{E}^{*}$ se transforment en opérateurs sur $\mathbf{C}[[z]]$ via l'isomorphisme $\Phi$. Plus précisément, si $U$ désigne un opérateur sur $\mathcal{E}^{*}$, il lui correspond l'opérateur $u$ sur $\mathbf{C}[[z]]$ défini par la relation

$$
\Phi U=u \Phi \Leftrightarrow u=\Phi U \Phi^{-1}
$$

que l'on appelle l'image de $U$. On a donc le diagramme:

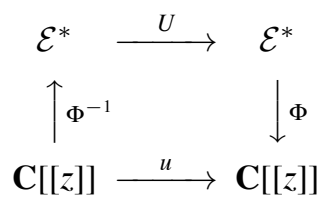

L'image de l'opérateur $I$ d'identité sur $\mathcal{E}^{*}$ est notée Id.

\section{EXEMPLE 1.}

a) La suite $\delta_{k}$ définie pour $k \geq 0$ et $n \geq 1$ par

$$
\delta_{k}(n)= \begin{cases}1 & \text { si } n=k+1 \\ 0 & \text { sinon }\end{cases}
$$

vérifie la relation

$$
\Phi\left(\delta_{k}\right)(z)=\frac{z^{k}}{k !} .
$$

On a $\delta_{0}:=(1,0,0, \ldots), \delta_{1}:=(0,1,0, \ldots)$, etc.

b) La suite $\mathbf{1}:=(1,1,1, \ldots)$ vérifie $\Phi(\mathbf{1})(z)=e^{z}$.

c) La suite $N:=(1,2,3, \ldots)$ vérifie la relation

$$
\Phi(N)(z)=\sum_{n \geq 0}(n+1) \frac{z^{n}}{n !}=z e^{z}+e^{z}=(1+z) e^{z} .
$$

d) Pour $\alpha \in \mathbf{C}$, la suite géométrique $\alpha^{N-1}:=\left(1, \alpha, \alpha^{2}, \alpha^{3}, \ldots\right)$ vérifie la relation

$$
\Phi\left(\alpha^{N-1}\right)(z)=\sum_{n \geq 0} \frac{\alpha^{n} z^{n}}{n !}=e^{\alpha z} .
$$

e) La suite $\frac{1}{N}:=\left(1, \frac{1}{2}, \frac{1}{3}, \frac{1}{4}, \ldots\right)$ vérifie la relation

$$
\Phi\left(\frac{1}{N}\right)(z)=\sum_{n \geq 0} \frac{1}{n+1} \frac{z^{n}}{n !}=\sum_{n \geq 0} \frac{z^{n}}{(n+1) !}=\frac{1}{z}\left(e^{z}-1\right) .
$$

Dans la suite de l'article, on désignera la suite $\frac{1}{N}$ sous le nom de suite harmonique. 
NotAtion. Si $a$ et $b$ sont deux suites dans $\mathcal{E}^{*}$, on note $a b$ la suite définie par

$$
(a b)(n)=a(n) b(n) .
$$

On a en particulier: $\mathbf{1} a=a$ et $\delta_{k} a=a(k+1) \delta_{k}$ pour tout $k \geq 0$. Muni de ce produit (appelé produit de Hadamard), $\mathcal{E}^{*}$ est une algèbre commutative, associative et unitaire notée $\mathcal{A}$. L'élément unité de $\mathcal{A}$ est la suite $\mathbf{1}$.

\subsection{LES OPÉRATEURS $L$ ET $R$}

DÉFINITION 3. L'opérateur $L$ de décalage à gauche sur $\mathcal{E}^{*}$ est défini par

$$
L(a)(n)=a(n+1),
$$

autrement dit

$$
(a(1), a(2), a(3), \ldots) \stackrel{L}{\longmapsto}(a(2), a(3), a(4), \ldots) .
$$

L'image de $L$ est l'opérateur de dérivation formelle $\partial$, car on a

$$
\Phi(L(a))(z)=\sum_{n \geq 0} a(n+2) \frac{z^{n}}{n !}=a(2)+a(3) z+a(4) \frac{z^{2}}{2 !}+\cdots=\partial \Phi(a)(z) .
$$

DÉFINITION 4. L'opérateur $R$ de décalage à droite sur $\mathcal{E}^{*}$ est défini par

$$
R(a)(n)= \begin{cases}a(n-1) & \text { si } n>1 \\ 0 & \text { si } n=1\end{cases}
$$

autrement dit

$$
(a(1), a(2), a(3), \ldots) \stackrel{R}{\longmapsto}(0, a(1), a(2), a(3), \ldots) .
$$

La suite $R(a)=(0, a(1), a(2), \ldots)$ est notée $(0, a)$. L'image de $R$ est l'opérateur d'intégration formelle $\int$, car on a

$$
\Phi(R(a))(z)=\sum_{n \geq 0} a(n+1) \frac{z^{n+1}}{(n+1) !}=a(1) z+a(2) \frac{z^{2}}{2 !}+\cdots=\int_{0}^{z} \Phi(a)(t) d t
$$

REMARQUE 1. On a la relation $L R=I$, mais on notera que $R L$ n'est pas l'identité:

$$
(a(1), a(2), a(3), \ldots) \stackrel{R L}{\longmapsto}(0, a(2), a(3), a(4), \ldots)
$$


2.3 Les opérateurs $D$ ET $S$

DÉfinition 5. Soit $V: \mathcal{E}^{*} \longrightarrow \mathbf{C}$ le morphisme d'évaluation défini par

$$
V(a)=a(1) .
$$

Son image est l'application $v: \mathbf{C}[[z]] \longrightarrow \mathbf{C}$ telle que $v(\Phi(a))=\Phi(a)(0)$.

L'opérateur $D: \mathcal{E}^{*} \longrightarrow \mathcal{E}^{*}$ est défini par

$$
D(a)(n)=V\left((I-L)^{n-1} a\right)=v\left((-\partial)^{n-1} \Phi(a)\right),
$$

c'est-à-dire

$$
D(a)(n)=\sum_{k=1}^{n}(-1)^{k-1}\left(\begin{array}{l}
n-1 \\
k-1
\end{array}\right) a(k) \quad \text { pour tout } n \geq 1,
$$

ou encore

$$
D(a)(n+1)=\sum_{k=0}^{n}(-1)^{k}\left(\begin{array}{l}
n \\
k
\end{array}\right) a(k+1) \quad \text { pour tout } n \geq 0 .
$$

On obtient ainsi

$$
\begin{aligned}
& D(a)(1)=a(1) \\
& D(a)(2)=a(1)-a(2) \\
& D(a)(3)=a(1)-2 a(2)+a(3) .
\end{aligned}
$$

Remarque 2. On définit dans [3] une version "continue" de l'opérateur $D$ dans un cadre différent.

Proposition 1 (Relation entre $D$ et la transformation binomiale). Soit $T$ la transformation binomiale définie sur $\mathcal{E}=\mathbf{C}^{\mathbf{N}}$ par

$$
T(a)(n)=\sum_{k=0}^{n}(-1)^{k}\left(\begin{array}{l}
n \\
k
\end{array}\right) a(k),
$$

et $\pi: \mathcal{E} \longrightarrow \mathcal{E}^{*}$ la projection naturelle :

$$
(a(0), a(1), a(2), a(3), \ldots) \stackrel{\pi}{\longmapsto}(a(1), a(2), a(3), \ldots) .
$$

On a la relation

$$
D\left(\frac{1}{N} \pi(a)\right)=a(0) \frac{1}{N}-\frac{1}{N} \pi(T(a))
$$


Démonstration. On a pour $n \geq 1$,

$$
\begin{aligned}
n D\left(\frac{1}{N} \pi(a)\right)(n) & =\sum_{k=1}^{n}(-1)^{k-1}\left(\begin{array}{l}
n-1 \\
k-1
\end{array}\right) \frac{n}{k} a(k) \\
& =-\sum_{k=1}^{n}(-1)^{k}\left(\begin{array}{l}
n \\
k
\end{array}\right) a(k)=-T(a)(n)+a(0) .
\end{aligned}
$$

Proposition 2 (Image de $D$ ). On a la relation

$$
\Phi(D(a))(z)=e^{z} \Phi(a)(-z),
$$

autrement dit, l'image $d$ de l'opérateur $D$ est telle que pour tout $f \in \mathbf{C}[[z]]$,

$$
d(f)(z)=e^{z} f(-z)
$$

Démonstration. On a

$$
\begin{aligned}
\Phi(D(a))(z) & =\sum_{n \geq 0} \sum_{k=0}^{n}\left(\begin{array}{l}
n \\
k
\end{array}\right)(-1)^{k} a(k+1) \frac{z^{n}}{n !} \\
& =\sum_{n \geq 0} \sum_{k=0}^{n}(-1)^{k} a(k+1) \frac{z^{k}}{k !} \frac{z^{n-k}}{(n-k) !} \\
& =\sum_{l \geq 0} \frac{z^{l}}{l !} \sum_{k \geq 0} a(k+1)(-1)^{k} \frac{z^{k}}{k !} \\
& =e^{z} \Phi(a)(-z) .
\end{aligned}
$$

COROLlaire 1. L'opérateur D est un automorphisme involutif, autrement dit,

$$
D=D^{-1}
$$

Démonstration. Pour montrer que $D=D^{-1}$, il suffit de montrer que $d=d^{-1}$. On a

$$
\begin{aligned}
d(f)=g \Longleftrightarrow e^{z} f(-z)=g(z) & \Longleftrightarrow f(-z)=e^{-z} g(z) \\
& \Longleftrightarrow f(z)=e^{z} g(-z) \Longleftrightarrow f=d(g) .
\end{aligned}
$$

EXEMPLE 2.

a) $\quad D(\mathbf{1})=\delta_{0}, D(N)=\delta_{0}-\delta_{1}, D\left(\delta_{1}\right)=\mathbf{1}-N$. 
b) On a vu que $\Phi\left(\alpha^{N-1}\right)=e^{\alpha z}$. Il en résulte par (2.2) que $D\left(\alpha^{N-1}\right)=$ $(1-\alpha)^{N-1}$. En particulier la suite $\left(\frac{1}{2}\right)^{N-1}$ est invariante par $D$.

c) On a vu que $\Phi\left(\frac{1}{N}\right)=\frac{1}{z}\left(e^{z}-1\right)$. Il en résulte par (2.2) que la suite harmonique est invariante par $D$ :

$$
D\left(\frac{1}{N}\right)=\frac{1}{N}
$$

Proposition 3. Pour toute suite $a$, on a

$$
D L(a)=(I-L) D(a)
$$

Démonstration. On a

$\Phi(D L(a))(z)=e^{z} \Phi(L(a))(-z)=e^{z} \partial \Phi(a)(-z)=e^{z} \Phi(a)(-z)-\partial\left(e^{z} \Phi(a)(-z)\right)$,

d'où $D L=D-L D=(I-L) D$.

DÉfINITION 6. L'opérateur de sommation $S: \mathcal{E}^{*} \longrightarrow \mathcal{E}^{*}$ est défini par

$$
S(a)(n)=\sum_{k=1}^{n} a(k) .
$$

EXEMPLE 3.

a) $\quad S\left(\delta_{0}\right)=\mathbf{1}, S(\mathbf{1})=N$.

b) $S\left(\alpha^{N-1}\right)=\frac{1}{1-\alpha}\left(\mathbf{1}-\alpha^{N}\right)$ pour $\alpha \neq 1$. En particulier,

$$
S\left((-1)^{N-1}\right)=\frac{1}{2}\left(\mathbf{1}+(-1)^{N-1}\right)=(1,0,1,0, \ldots) .
$$

PROPOSITION 4. L'opérateur $S$ est un automorphisme d'inverse

$$
S^{-1}=I-R
$$

Démonstration. On a

$$
b(n)=S(a)(n) \Leftrightarrow a(n)=b(n)-b(n-1)
$$

pour $n>1$ et $a(1)=b(1)$. 
Notation. On pose $H:=S\left(\frac{1}{N}\right), O:=S\left(\frac{1}{2 N-1}\right)$, et pour $k \geq 2$, $H^{(k)}:=S\left(\frac{1}{N^{k}}\right)$ et $O^{(k)}:=S\left(\frac{1}{(2 N-1)^{k}}\right)$ avec:

$$
\frac{1}{N^{k}}:=\underbrace{\frac{1}{N} \cdots \frac{1}{N}}_{k} \quad \text { et } \quad \frac{1}{(2 N-1)^{k}}:=\underbrace{\frac{1}{2 N-1} \cdots \frac{1}{2 N-1}}_{k} \text {. }
$$

Pour $n \geq 1$, on a donc

$$
\begin{aligned}
H(n) & =\sum_{k=1}^{n} \frac{1}{k}, & O(n) & =\sum_{k=1}^{n} \frac{1}{2 k-1}, \\
H^{(2)}(n) & =\sum_{k=1}^{n} \frac{1}{k^{2}}, & O^{(2)}(n) & =\sum_{k=1}^{n} \frac{1}{(2 k-1)^{2}} .
\end{aligned}
$$

EXEMPLE 4. Les relations

$$
\sum_{k=1}^{n} H(k)=(n+1) H(n)-n \quad \text { et } \quad \sum_{k=1}^{n} \frac{H(k)}{k}=\frac{1}{2}(H(n))^{2}+\frac{1}{2} H^{(2)}(n)
$$

se démontrent facilement par récurrence; elles se traduisent par

$$
S(H)=(N+1) H-N \quad \text { et } \quad S\left(\frac{1}{N} H\right)=\frac{1}{2}\left(H^{2}+H^{(2)}\right) .
$$

PROPOSITION 5. On a la relation

$$
\Phi(S(a))(z)=\Phi(a)(z)-e^{z} \int_{0}^{-z} e^{t} \Phi(a)(-t) d t
$$

Autrement dit, l'image $s$ de $S$ est l'opérateur $I d-d \int d$.

Démonstration. On a la relation $(L-I) S=L$ qui se traduit par

$$
(\partial-\mathrm{Id}) \Phi(S(a))=\partial \Phi(a) \text {. }
$$

En résolvant l'équation différentielle $(\partial-\mathrm{Id}) \Phi(S(a))=\partial \Phi(a)$, on obtient

$$
\begin{aligned}
\Phi(S(a))(z) & =\Phi(a)(z)+e^{z} \int_{0}^{z} e^{-t} \Phi(a)(t) d t \\
& =\Phi(a)(z)-e^{z} \int_{0}^{-z} e^{t} \Phi(a)(-t) d t
\end{aligned}
$$


PROPOSITION 6. Pour tout entier naturel $p$, l'automorphisme DS ${ }^{p}$ est involutif:

$$
D S^{p}=S^{-p} D=\left(D S^{p}\right)^{-1}
$$

En particulier,

$$
D S=S^{-1} D=(I-R) D
$$

Démonstration. Le cas $p=0$ traduit l'involutivité de $D$. On a vu que l'image $s$ de $S$ est l'opérateur Id $-d \int d$. On en déduit que

$$
S=I-D R D \text {. }
$$

D'où $D S=D-R D=(I-R) D=S^{-1} D=(D S)^{-1}$. On procède alors par récurrence sur $p \geq 1$ en écrivant que $D S^{p+1}=D S^{p} S=S^{-p} D S=S^{-p} S^{-1} D=$ $S^{-(p+1)} D$.

EXEMPLE 5. Comme $a=\frac{1}{N}$ est invariante par $D$, on en déduit que

$$
D(H)=(I-R)\left(\frac{1}{N}\right)=\frac{1}{N}+\left(0,-\frac{1}{N}\right)=\delta_{0}+\left(0,-\frac{1}{N(N+1)}\right),
$$

c'est-à-dire

$$
D(H)(n)= \begin{cases}-\frac{1}{n(n-1)} & \text { si } n>1 \\ 1 & \text { si } n=1\end{cases}
$$

PROPOSITION 7. Pour toute suite $a \in \mathcal{E}^{*}$, on a la relation

$$
D\left(\frac{1}{N} S(a)\right)=\frac{1}{N} D(a) \text {. }
$$

Démonstration. Comme $S^{-1}=I-R$, on a $s^{-1}=\mathrm{Id}-\int$, d'où

$$
\begin{aligned}
\Phi\left(S^{-1}(a)\right)(z)=\Phi(a)(z)-\int_{0}^{z} \Phi(a)(t) d t & =\Phi(a)(z)-z \sum_{n \geq 0} \frac{a(n+1)}{n+1} \frac{z^{n}}{n !} \\
& =\Phi(a)(z)-z \Phi\left(\frac{1}{N} a\right) .
\end{aligned}
$$

En remplaçant $a$ par $S(a)$ dans la relation précédente, on obtient alors l'égalité

$$
\Phi\left(\frac{1}{N} S(a)\right)=\frac{\Phi(S(a))-\Phi(a)}{z} .
$$

D'après (2.4), on a donc 


$$
\Phi\left(\frac{1}{N} S(a)\right)=-\frac{e^{z}}{z} \int_{0}^{-z} e^{t} \Phi(a)(-t) d t .
$$

D'où

$$
\begin{aligned}
\Phi\left(D\left(\frac{1}{N} S(a)\right)\right) & =\frac{1}{z} \int_{0}^{z} e^{t} \Phi(a)(-t) d t \\
& =\frac{1}{z} \int_{0}^{z} \Phi(D(a))(t) d t=\Phi\left(\frac{1}{N} D(a)\right)
\end{aligned}
$$

EXEMPLE 6. Par (2.5) appliquée à la suite $\frac{1}{N}$, on déduit

$$
D\left(\frac{1}{N} H\right)=\frac{1}{N} D\left(\frac{1}{N}\right)=\frac{1}{N} \frac{1}{N}=\frac{1}{N^{2}},
$$

d'où aussi

$$
D\left(\frac{1}{N} H^{(2)}\right)=\frac{1}{N} D\left(\frac{1}{N^{2}}\right)=\frac{1}{N} \frac{1}{N} H=\frac{1}{N^{2}} H .
$$

\subsection{FORMULE DE VANDERMONDE}

Notation. Pour $\alpha \in \mathbf{C}$, on note $(\alpha)_{0}=1$ et pour $n \geq 1$,

$$
(\alpha)_{n}=\alpha(\alpha+1) \ldots(\alpha+n-1) .
$$

On note $(\alpha)_{N}$ la suite $n \mapsto(\alpha)_{n}$. On pose $N !:=(1)_{N}$.

Proposition 8. Pour $\alpha \in \mathbf{C}$ et pour $\beta \in \mathbf{R}-\{0,-1,-2, \ldots\}$, on a la relation

$$
D\left(\frac{1}{N} \frac{(\alpha)_{N}}{(\beta)_{N}}\right)=\frac{1}{N}-\frac{1}{N} \frac{(\beta-\alpha)_{N}}{(\beta)_{N}} .
$$

En particulier, pour $\alpha \in \mathbf{R}-\{0,-1,-2, \ldots\}$,

$$
D\left(\frac{(N-1) !}{(\alpha)_{N}}\right)=\frac{1}{N}-\frac{(\alpha-1)_{N}}{N(\alpha)_{N}}=\frac{1}{N+\alpha-1} .
$$

Démonstration. D'après la formule de Vandermonde (cf. [7], p.25), on peut écrire

$$
\sum_{k=0}^{n}(-1)^{k}\left(\begin{array}{l}
n \\
k
\end{array}\right) \frac{(\alpha)_{k}}{(\beta)_{k}}=\sum_{k=0}^{n} \frac{(\alpha)_{k}(-n)_{k}}{(\beta)_{k} k !}=\frac{(\beta-\alpha)_{n}}{(\beta)_{n}} .
$$

La relation (2.6) s'en déduit alors par (2.1). 
REMARQUE 3. La formule de Vandermonde peut s'écrire plus simplement

$$
D\left(\frac{(\alpha)_{N-1}}{(\beta)_{N-1}}\right)=\frac{(\beta-\alpha)_{N-1}}{(\beta)_{N-1}}
$$

EXEMPLE 7. En appliquant (2.7) avec $\alpha=\frac{1}{2}$, il vient

$$
D\left(\frac{2^{2 x}(N !)^{2}}{N(2 N) !}\right)=\frac{1}{N}-\frac{1}{N} \frac{-1 / 2}{N-1 / 2}=\frac{2}{2 N-1} \text {. }
$$

En posant

$$
\left(\begin{array}{c}
2 N \\
N
\end{array}\right):=\frac{(2 N) !}{(N !)^{2}}
$$

on en déduit

$$
D\left(\frac{1}{2 N-1}\right)=\frac{1}{N} \frac{2^{2 N-1}}{\left(\begin{array}{c}
2 N \\
N
\end{array}\right)}
$$

d'où aussi par (2.5):

$$
D\left(\frac{1}{N} O\right)=\frac{1}{N} D\left(\frac{1}{2 N-1}\right)=\frac{1}{N^{2}} \frac{2^{2 N-1}}{\left(\begin{array}{c}
2 N \\
N
\end{array}\right)} .
$$

\section{LE PRODUIT HARMONIQUE}

3.1 L'ALGÈBRE $\mathcal{H}=\left(\mathcal{E}^{*}, \bowtie\right)$

On rappelle que $\mathcal{A}$ désigne l'algèbre $\left(\mathcal{E}^{*}, \cdot\right)$ munie du produit de Hadamard des suites.

DÉFINITION 7. On définit le produit harmonique $a \bowtie b$ de deux suites $a$ et $b$ dans $\mathcal{E}^{*}$ par

$$
a \bowtie b:=D(D(a) D(b)) .
$$

Comme $D=D^{-1}$, on déduit immédiatement de la définition précédente les deux relations fondamentales suivantes:

$$
D(a \bowtie b)=D(a) D(b),
$$

et

$$
D(a b)=D(a) \bowtie D(b) .
$$


EXEMPLE 8.

a) On a $\mathbf{1} \bowtie a=a(1) \mathbf{1}$, car

$$
D(\mathbf{1} \bowtie a)=D(\mathbf{1}) D(a)=\delta_{0} D(a)=D(a)(1) \delta_{0}=a(1) \delta_{0}=a(1) D(\mathbf{1}) .
$$

b) On a $N \bowtie a=a(2) \mathbf{1}+(a(1)-a(2)) N$, car

$$
\begin{aligned}
D(N) D(a)=\left(\delta_{0}-\delta_{1}\right) D(a) & =D(a)(1) \delta_{0}-D(a)(2) \delta_{1} \\
& =a(1) D(N)+a(2) D(1-N) .
\end{aligned}
$$

c) On a $\alpha^{N-1} \bowtie \beta^{N-1}=(\alpha+\beta-\alpha \beta)^{N-1}$, car

$$
\begin{aligned}
D\left(\alpha^{N-1} \bowtie \beta^{N-1}\right) & =(1-\alpha)^{N-1}(1-\beta)^{N-1} \\
& =(1-(\alpha+\beta-\alpha \beta))^{N-1} \\
& =D\left((\alpha+\beta-\alpha \beta)^{N-1}\right) .
\end{aligned}
$$

d) Enfin, par la formule de Vandermonde (2.8), on a

$$
\frac{(\gamma-\beta)_{N-1}}{(\gamma)_{N-1}} \bowtie \frac{(\beta-\alpha)_{N-1}}{(\beta)_{N-1}}=\frac{(\gamma-\alpha)_{N-1}}{(\gamma)_{N-1}} .
$$

Proposition 9. L'espace $\left(\mathcal{E}^{*}, \bowtie\right)$ est une $\mathbf{C}$-algèbre commutative, associative et unitaire notée $\mathcal{H}$, isomorphe à l'algèbre $\mathcal{A}$. L'élément unité dans $\mathcal{H}$ est la suite $\delta_{0}$.

Démonstration. La bilinéarité du produit $\bowtie$ résulte de la linéarité de $D$ et de la bilinéarité du produit de Hadamard. De plus, il résulte immédiatement des propriétés (3.1) et (3.2) que l'opérateur $D$ réalise un isomorphisme d'algèbre entre les $\mathbf{C}$-algèbres $\mathcal{A}$ et $\mathcal{H}$.

Il en résulte que $\mathcal{H}$ hérite des propriétés d'associativité et de commutativité de $\mathcal{A}$. En particulier, l'élément unité de $\mathcal{H}$ est l'image de 1 par $D$, c'est-àdire $\delta_{0}$.

REMARQUe 4. L'algèbre $\mathcal{H}$ contient des diviseurs de zéro. On a par exemple

$$
\mathbf{1} \delta_{1}=0 .
$$

COROLlaire 2. Une suite a est inversible dans $\mathcal{H}$ si et seulement si la suite $D(a)$ est inversible dans $\mathcal{A}$ (i.e. $D(a)(n) \neq 0$ pour tout $n)$. Dans ce cas, l'inverse harmonique de a est donné par la formule

$$
a^{\bowtie(-1)}=D\left(\frac{1}{D(a)}\right) .
$$


Démonstration.

$$
a \bowtie b=\delta_{0} \quad \Leftrightarrow \quad D(a) D(b)=D\left(\delta_{0}\right)=\mathbf{1} \quad \Leftrightarrow \quad D(b)=\frac{1}{D(a)} .
$$

EXEMPLE 9.

a) Les suites $\mathbf{1}$ et $N$ ne sont pas inversibles dans $\mathcal{H}$.

b) $\left(\frac{1}{N}\right)^{\bowtie(-1)}=D(N)=\delta_{0}-\delta_{1}$,

c) $\left(\alpha^{N-1}\right)^{\bowtie(-1)}=\left(\frac{\alpha}{\alpha-1}\right)^{N-1}$.

\subsection{PUISSANCES HARMONIQUES $k$-IÈMES}

DÉFInITION 8. Pour toute suite $a \in \mathcal{E}^{*}$, on définit pour tout entier $k \geq 0$, la puissance harmonique $k$-ième de $a$ notée $a^{\bowtie k}$ par

$$
a^{\bowtie 0}=\delta_{0} \quad \text { et } a^{\bowtie(k+1)}=a^{\bowtie k} \bowtie a .
$$

Par récurrence sur $k$, on en déduit immédiatement la formule suivante:

$$
a^{\bowtie k}=D(\underbrace{D(a) \ldots D(a)}_{k})=D\left((D(a))^{k}\right) .
$$

En particulier, si $a$ est invariante par $D$, alors $a^{\bowtie k}=D\left(a^{k}\right)$.

EXEMPLE 10.

a) $\left(\frac{1}{N}\right)^{\bowtie k}=D\left(\frac{1}{N^{k}}\right)$.

b) $\quad N^{\bowtie k}=D\left(\left(\delta_{0}-\delta_{1}\right)^{k}\right)=\mathbf{1}+(-1)^{k}(1-N)= \begin{cases}N & \text { si } k \text { est impair }, \\ 2-N & \text { si } k \text { est pair. }\end{cases}$

c) Soient les nombres de Stirling de deuxième espèce

$$
S(k, n)=\frac{1}{n !} \sum_{m=0}^{n}(-1)^{m-k}\left(\begin{array}{l}
n \\
m
\end{array}\right) m^{k}
$$

On a

$$
\left(\delta_{1}\right)^{\bowtie k}=\sum_{n=0}^{k} n ! S(k, n) \delta_{n},
$$

car

$$
\left(\delta_{1}\right)^{\bowtie k}=D\left((-1)^{k}(N-1)^{k}\right)
$$


et

$$
D\left((-1)^{k}(N-1)^{k}\right)(n+1)=\sum_{m=0}^{n}(-1)^{m-k}\left(\begin{array}{l}
n \\
m
\end{array}\right) m^{k}=n ! S(k, n)
$$

3.3 IMAGE DE $\mathcal{H}$ DANS $\mathbf{C}[[z]]$

THÉORÈME 1. Pour toutes suites a et $b$, on pose

$$
(\Phi(a) \otimes \Phi(b))(x, y):=\Phi(a)(x) \Phi(b)(y) .
$$

On a alors

$$
\Phi(a \bowtie b)(z)=\sum_{n \geq 0}\left(v_{x} \otimes v_{y}\right)\left(\partial_{x}+\partial_{y}-\partial_{x} \partial_{y}\right)^{n}(\Phi(a) \otimes \Phi(b)) \frac{z^{n}}{n !} .
$$

Il en résulte que pour tout entier $n \geq 0$,

$$
(a \bowtie b)(n+1)=\sum_{\substack{0 \leq k \leq n \\ 0 \leq l \leq n}} C_{n}^{k, l} a(k+1) b(l+1),
$$

où les nombres $C_{n}^{k, l}$ sont définis par l'identité

$$
(X+Y-X Y)^{n}=\sum_{\substack{0 \leq k \leq n \\ 0 \leq l \leq n}} C_{n}^{k, l} X^{k} Y^{l} .
$$

Démonstration. On a

$$
\Phi(a \bowtie b)(z)=\Phi(D(D(a) D(b)))(z)=e^{z} \Phi(D(a) D(b))(-z)
$$

et

$$
D(a)(n+1)=v\left((\operatorname{Id}-\partial)^{n} \Phi(a)\right) \quad \text { avec } \quad v(\Phi(a))=\Phi(a)(0)=a(1) .
$$

D'où

$$
\begin{aligned}
(D(a) D(b))(n+1) & =\left(v_{x} \otimes v_{y}\right)\left[\left(\operatorname{Id}-\partial_{x}\right)\left(\operatorname{Id}-\partial_{y}\right)\right]^{n}(\Phi(a) \otimes \Phi(b))(x, y) \\
& =\left(v_{x} \otimes v_{y}\right)\left[\operatorname{Id}-\left(\partial_{x}+\partial_{y}-\partial_{x} \partial_{y}\right)\right]^{n}(\Phi(a) \otimes \Phi(b))(x, y) .
\end{aligned}
$$

On en déduit que

$$
\begin{aligned}
\Phi(D(a) D(b))(-z) & =\left(v_{x} \otimes v_{y}\right) \sum_{n \geq 0}\left[\operatorname{Id}-\left(\partial_{x}+\partial_{y}-\partial_{x} \partial_{y}\right)\right]^{n}(\Phi(a) \otimes \Phi(b))(-1)^{n} \frac{z^{n}}{n !} \\
& =e^{-z}\left(v_{x} \otimes v_{y}\right) e^{\left(\partial_{x}+\partial_{y}-\partial_{x} \partial_{y}\right) z}(\Phi(a) \otimes \Phi(b)) .
\end{aligned}
$$


Il en résulte que

$$
\begin{aligned}
\Phi(a \bowtie b)(z) & =\left(v_{x} \otimes v_{y}\right) e^{\left(\partial_{x}+\partial_{y}-\partial_{x} \partial_{y}\right) z} \Phi(a) \otimes \Phi(b) \\
& =\sum_{n \geq 0}\left(v_{x} \otimes v_{y}\right)\left(\partial_{x}+\partial_{y}-\partial_{x} \partial_{y}\right)^{n}(\Phi(a) \otimes \Phi(b)) \frac{z^{n}}{n !} .
\end{aligned}
$$

Par identification du terme général, on en déduit que

$$
\begin{aligned}
(a \bowtie b)(n+1) & =\left(v_{x} \otimes v_{y}\right)\left(\partial_{x}+\partial_{y}-\partial_{x} \partial_{y}\right)^{n}(\Phi(a) \otimes \Phi(b)) \\
& =\sum_{\substack{0 \leq k \leq n \\
0 \leq l \leq n}}\left(v_{x} \otimes v_{y}\right) C_{n}^{k, l} \partial_{x}^{k} \Phi(a) \partial_{y}^{l} \Phi(b) \\
& =\sum_{\substack{0 \leq k \leq n \\
0 \leq l \leq n}} C_{n}^{k, l} a(k+1) b(l+1)
\end{aligned}
$$

avec

$$
(X+Y-X Y)^{n}=\sum_{\substack{0 \leq k \leq n \\ 0 \leq l \leq n}} C_{n}^{k, l} X^{k} Y^{l}
$$

COROLlaIRE 3 (Expression explicite du produit harmonique).

$$
(a \bowtie b)(n+1)=\sum_{0 \leq l \leq k \leq n}(-1)^{k-l}\left(\begin{array}{l}
n \\
k
\end{array}\right)\left(\begin{array}{l}
k \\
l
\end{array}\right) a(k+1) b(n+1-l) \quad(n \geq 0) .
$$

Démonstration. En développant $(X+Y-X Y)^{n}$ par la formule du binôme et en identifiant le coefficient de $X^{k} Y^{l}$, on vérifie que

$C_{n}^{k, l}=(-1)^{k+l-n} \frac{n !}{(n-k) !(n-l) !(l+k-n) !} \quad$ si $n \leq k+l, \quad$ et $C_{n}^{k, l}=0$ sinon,

d'où

$$
\begin{aligned}
(a \bowtie b)(n+1) & =\sum_{\substack{0 \leq k \leq n \\
0 \leq l \leq n}} C_{n}^{k, l} a(k+1) b(l+1) \\
& =\sum_{\substack{0 \leq k \leq n \\
0 \leq l \leq n \\
k+l \geq n}}(-1)^{k+l-n} \frac{n !}{(n-k) !(n-l) !(l+k-n) !} a(k+1) b(l+1) \\
& =\sum_{0 \leq l \leq k \leq n}(-1)^{k-l}\left(\begin{array}{l}
n \\
k
\end{array}\right)\left(\begin{array}{l}
k \\
l
\end{array}\right) a(k+1) b(n-l+1) .
\end{aligned}
$$


COROLlaire 4. On a la relation

$$
\Phi(a \bowtie b)(z)=v_{y}\left(\Phi(a)\left[\left(\operatorname{Id}-\partial_{y}\right) z\right] \Phi(b)[y+z]\right) .
$$

Il en résulte que

$$
\Phi\left(\alpha^{N-1} \bowtie a\right)(z)=e^{\alpha z} \Phi(a)((1-\alpha) z),
$$

ce qui se traduit par l'identité

$$
\left(\alpha^{N-1} \bowtie a\right)(n+1)=\alpha^{n} \sum_{k=0}^{n}\left(\begin{array}{l}
n \\
k
\end{array}\right)\left(\frac{1-\alpha}{\alpha}\right)^{k} a(k+1) .
$$

Démonstration. On a

$$
e^{\left(\partial_{x}+\partial_{y}-\partial_{x} \partial_{y}\right) z} \Phi(a)=e^{\partial_{x}\left(\mathrm{Id}-\partial_{y}\right) z} \Phi(a) e^{\left(\partial_{y}\right) z}=\Phi(a)\left[x+\left(\mathrm{Id}-\partial_{y}\right) z\right] e^{\left(\partial_{y}\right) z}
$$

donc

$$
\begin{aligned}
\Phi(a \bowtie b)(z) & =\left(v_{x} \otimes v_{y}\right) \Phi(a)\left[x+\left(\mathrm{Id}-\partial_{y}\right) z\right] e^{\left(\partial_{y}\right) z} \Phi(b) \\
& =\left(v_{x} \otimes v_{y}\right) \Phi(a)\left[x+\left(\mathrm{Id}-\partial_{y}\right) z\right] \Phi(b)[y+z] \\
& =v_{y}\left(\Phi(a)\left[\left(\mathrm{Id}-\partial_{y}\right) z\right] \Phi(b)[y+z]\right) .
\end{aligned}
$$

On a vu que $\Phi\left(\alpha^{x-1}\right)(z)=e^{\alpha z}$ donc

$$
\begin{aligned}
\Phi\left(\alpha^{x-1} \bowtie a\right)(z) & =v_{y}\left(\Phi\left(\alpha^{x-1}\right)\left[\left(\operatorname{Id}-\partial_{y}\right) z\right] \Phi(a)[y+z]\right) \\
& =v_{y}\left(e^{\alpha\left(\operatorname{Id}-\partial_{y}\right) z} \Phi(a)[y+z]\right) \\
& =e^{\alpha z} v_{y}\left(e^{-\alpha z \partial_{y}} \Phi(a)[y+z]\right) \\
& =e^{\alpha z} \sum_{n \geq 0} \frac{(-\alpha z)^{n}}{n !} \partial^{n} \Phi(a)(z) \\
& =e^{\alpha z} \Phi(a)((1-\alpha) z) .
\end{aligned}
$$

EXEMPLE 11.

$$
\left(\left(\frac{1}{2}\right)^{N-1} \bowtie a\right)(n+1)=\frac{1}{2^{n}} \sum_{k=0}^{n}\left(\begin{array}{l}
n \\
k
\end{array}\right) a(k+1) .
$$

PROPOSITION 10 (Caractérisation des suites invariantes par D). Une suite $a \in \mathcal{E}^{*}$ est invariante par $D$ si et seulement si elle peut s'écrire sous la forme

$$
a=\left(\frac{1}{2}\right)^{N-1} \bowtie b,
$$

où la suite $b \in \mathcal{E}^{*}$ est telle que $b(2 k)=0$ pour tout $k \geq 1$. 
Démonstration. On a

$$
D(a)=a \Leftrightarrow \Phi(D(a))=\Phi(a) \Leftrightarrow e^{z} \Phi(a)(-z)=\Phi(a)(z) .
$$

Posons $\phi(z)=e^{-\frac{z}{2}} \Phi(a(z))$. On a donc $D(a)=a \Leftrightarrow \phi(z)=\phi(-z)$. Dans ce cas, $\phi$ peut toujours s'écrire

$$
\phi(z)=\Phi(b)\left(\frac{z}{2}\right) \quad \text { avec } b(2 n)=0 \text { pour } n \geq 1,
$$

et on a alors

$$
\Phi(a(z))=e^{\frac{z}{2}} \phi(z)=e^{\frac{z}{2}} \Phi(b)\left(\frac{z}{2}\right)=\Phi\left(\left(\frac{1}{2}\right)^{N-1} \bowtie b\right)(z) .
$$

EXEMPLE 12.

a) La suite harmonique s'écrit

$$
\frac{1}{N}=\left(\frac{1}{2}\right)^{N-1} \bowtie b
$$

avec $b=\frac{1}{N} \bowtie(-1)^{N-1}=\left(1,0, \frac{1}{3}, 0, \frac{1}{5}, \ldots\right)$.

b) La suite

$$
a=\frac{1}{2}\left(\delta_{0}+\mathbf{1}\right)=\left(1, \frac{1}{2}, \frac{1}{2}, \frac{1}{2}, \ldots\right)
$$

est invariante par $D$. Elle s'écrit

$$
a=\left(\frac{1}{2}\right)^{N-1} \bowtie(1,0,1,0, \ldots) .
$$

REMARQUE 5. On comparera le critère d'invariance précédent avec celui donné par Sun ([11] Corollary 3.3 (a)).

REMARQue 6 (Sommation d'Euler des séries). Pour $q>0$, on définit la suite $a^{(q)}$ par

$$
a^{(q)}(n+1)=\frac{1}{(q+1)^{n}} \sum_{k=0}^{n}\left(\begin{array}{l}
n \\
k
\end{array}\right) q^{n-k} a(k+1) \quad(n \geq 0) .
$$

D'après [6], la série $\sum_{n \geq 1} a(n)$ est dite $(\mathrm{E}, q)$ sommable si la série $\sum_{n \geq 1} a^{(q)}(n)$ converge; on pose alors

$$
\sum_{n \geq 1}^{(\mathrm{E}, q)} a(n):=\frac{1}{q+1} \sum_{n=0}^{\infty} a^{(q)}(n+1) .
$$


D'après (3.7), on a l'interprétation suivante de $a^{(q)}$ :

$$
a^{(q)}=\alpha^{N-1} \bowtie a \quad \text { avec } \quad \alpha=\frac{q}{q+1} .
$$

On obtient ainsi une reformulation du théorème de Hardy ([6], p. 178-179):

THÉORÈME (Hardy). Si la série $\sum_{n \geq 1} a(n)$ est convergente alors elle est $(\mathrm{E}, q)$ sommable et on a

$$
\frac{1}{q+1} \sum_{n=1}^{+\infty}\left(\left(\frac{q}{q+1}\right)^{N-1} \bowtie a\right)(n)=\sum_{n=1}^{+\infty} a(n) .
$$

\subsection{HARMONICITÉ}

THÉORÈME 2. Pour toute suite $a \in \mathcal{E}^{*}$, on a la relation

$$
\frac{1}{N} \bowtie a=\frac{1}{N} S(a)
$$

Démonstration. Il suffit de montrer que $D\left(\frac{1}{N} \bowtie a\right)=D\left(\frac{1}{N} S(a)\right)$. Or, par (2.5), on a $D\left(\frac{1}{N} S(a)\right)=\frac{1}{N} D(a)=D\left(\frac{1}{N}\right) D(a)=D\left(\frac{1}{N} \bowtie a\right)$.

COROllaire 5. Pour tout entier $k \geq 1$,

$$
D\left(\frac{1}{N^{k+1}}\right)=\frac{1}{N} S D\left(\frac{1}{N^{k}}\right) .
$$

Démonstration.

$$
\begin{aligned}
D\left(\frac{1}{N^{k+1}}\right) & =\left(\frac{1}{N}\right)^{\bowtie(k+1)}=\frac{1}{N} \bowtie\left(\frac{1}{N}\right)^{\bowtie k} \\
& =\frac{1}{N} S\left(\left(\frac{1}{N}\right)^{\bowtie k}\right)=\frac{1}{N} S D\left(\frac{1}{N^{k}}\right) .
\end{aligned}
$$

EXEMPLE 13.

$$
\begin{gathered}
\frac{1}{N} \bowtie \frac{1}{N}=D\left(\frac{1}{N^{2}}\right)=\frac{1}{N} H, \\
\left(\frac{1}{N}\right)^{\bowtie 3}=D\left(\frac{1}{N^{3}}\right)=\frac{1}{N} S\left(\frac{1}{N} H\right)=\frac{1}{2 N}\left(H^{2}+H^{(2)}\right) .
\end{gathered}
$$


Notation. Pour tout $p \in \mathbf{R}-\{-1,-2, \ldots\}$, on note $p !=\Gamma(p+1)$; on note $\Gamma(N+p)$ la suite $n \mapsto \Gamma(n+p)$. On pose

$$
(N)_{p}:=\frac{\Gamma(N+p)}{\Gamma(N)} .
$$

Pour $p$ entier naturel, on a $(N)_{0}=\mathbf{1}$, et pour $p \geq 1$

$$
(N)_{p}=N(N+1) \cdots(N+p-1) .
$$

Le théorème 2 se généralise alors de la manière suivante:

THÉORÈME 3. Pour toute suite $a \in \mathcal{E}^{*}$ et tout réel $p \neq-1,-2,-3, \ldots$, on a la relation

$$
\frac{p !}{(N)_{p+1}} \bowtie a=\frac{p !}{(N)_{p+1}} S\left(\frac{(N)_{p}}{p !} a\right),
$$

ce qui, pour $p$ entier $\geq 0$, se traduit par

$$
\left(\frac{p !}{N(N+1) \ldots(N+p)} \bowtie a\right)(n)=\frac{p !}{n(n+1) \ldots(n+p)} \sum_{k=1}^{n} \frac{k(k+1) \ldots(k+p-1)}{p !} a(k) .
$$

Démonstration. En appliquant (2.7) avec $p=\alpha-1$, on obtient

$$
D\left(\frac{1}{N+p}\right)=\frac{(N-1) !}{(p+1)_{N}}=\frac{\Gamma(N) \Gamma(p+1)}{\Gamma(N+p+1)}=\frac{p !}{(N)_{p+1}},
$$

par conséquent,

$$
\frac{p !}{(N)_{p+1}} \bowtie a=D\left(\frac{1}{N+p} D(a)\right) .
$$

Posons alors

$$
f_{p+1}(z)=\Phi\left(D\left(\frac{1}{N+p} D(a)\right)\right)(z)
$$

On a

$$
f_{p+1}(z)=e^{z} \sum_{n \geq 0}(D(a))(n+1) \frac{1}{n+1+p} \frac{(-z)^{n}}{n !}
$$

et donc

$$
e^{-z} z^{p+1} f_{p+1}(z)=\sum_{n \geq 0}(D(a))(n+1)(-1)^{n} \frac{1}{n+1+p} \frac{z^{n+p+1}}{n !} .
$$


Par conséquent,

$$
\begin{aligned}
e^{-z} z^{p+1} f_{p+1}(z) & =\int_{0}^{z} t^{p} \sum_{n \geq 0}(D(a))(n+1)(-1)^{n} \frac{t^{n}}{n !} d t \\
& =\int_{0}^{z} e^{-t} t^{p} \sum_{n \geq 0} a(n+1) \frac{t^{n}}{n !} d t \\
& =\int_{0}^{z} e^{-t} t^{p} \Phi(a)(t) d t
\end{aligned}
$$

On obtient donc

$$
f_{p+1}(z)=e^{z} \frac{1}{z^{p+1}} \int_{0}^{z} e^{-t} t^{p} \Phi(a)(t) d t .
$$

Par le changement de variable $u=t z$, on a aussi

$$
\begin{aligned}
f_{p+1}(z) & =\int_{0}^{1} e^{z(1-u)} u^{p} \Phi(a)(u z) d u \\
& =\sum_{k, l} \frac{z^{k}}{k !} \frac{z^{l}}{l !} a(k+1) \int_{0}^{1}(1-u)^{l} u^{p+k} d u \\
& =\sum_{k, l} \frac{z^{k}}{k !} \frac{z^{l}}{l !} a(k+1) \frac{l !(p+k) !}{(p+k+l+1) !} \\
& =\sum_{n} \frac{z^{n}}{n !} \sum_{k+l=n} \frac{n !}{k ! l !} a(k+1) \frac{l !(p+k) !}{(p+k+l+1) !} \\
& =\sum_{n} \frac{z^{n}}{n !} \sum_{k=0}^{n} \frac{n !}{k !} \frac{(p+k) !}{(p+n+1) !} a(k+1) .
\end{aligned}
$$

Or,

$\sum_{k=0}^{n} \frac{n !}{k !} \frac{(p+k) !}{(p+n+1) !} a(k+1)=\frac{1}{(n+1) \ldots(n+1+p)} \sum_{k=1}^{n+1} k(k+1) \ldots(k+p-1) a(k)$

ce qui montre que

$\Phi\left(\frac{p !}{(N)_{p+1}} \bowtie a\right)=\Phi\left(\frac{1}{(N)_{p+1}} S\left((N)_{p} a\right)\right)=\Phi\left(\frac{p !}{(N)_{p+1}} S\left(\frac{(N)_{p}}{p !} a\right)\right)$.

EXEMPLE 14. Pour $p=1$, on a

$$
\frac{1}{N(N+1)} \bowtie a=\frac{1}{N(N+1)} S(N a)
$$


c'est-à-dire

$$
\left(\frac{1}{N(N+1)} \bowtie a\right)(n)=\frac{1}{n(n+1)} \sum_{k=1}^{n} k a(k) .
$$

On en déduit que

$$
\left(\frac{1}{N+1} \bowtie a\right)(n)=\frac{1}{n(n+1)} \sum_{k=1}^{n}(n+1-k) a(k) .
$$

Corollaire 6. Pour toute suite $a \in \mathcal{E}^{*}$ et pour tout entier $p \geq 0$,

$$
D\left(\frac{1}{(N)_{p+1}} S(a)\right)=\frac{1}{(N)_{p+1}} S^{p}(D(a)) .
$$

Démonstration. Par récurrence sur $p$. Pour $p=0$, c'est la formule (2.5). En écrivant $(N)_{p+1}=(N+p)(N)_{p}$, on obtient

$$
\begin{aligned}
D\left(\frac{1}{(N)_{p+1}} S(a)\right) & =D\left(\frac{1}{N+p}\right) \bowtie D\left(\frac{1}{(N)_{p}} S(a)\right) \\
& =\frac{p !}{(N)_{p+1}} \bowtie \frac{1}{(N)_{p}} S^{p-1}(D(a)) \\
& =\frac{p !}{(N)_{p+1}} S\left(\frac{(N)_{p}}{p !} \frac{1}{(N)_{p}} S^{p-1}(D(a))\right) \\
& =\frac{1}{(N)_{p+1}} S^{p}(D(a)) .
\end{aligned}
$$

EXEMPLE 15.

a) Pour $a=\frac{1}{N}$ et $p=1$,

$$
D\left(\frac{H}{N(N+1)}\right)=\frac{H}{N(N+1)} .
$$

b) Pour $a=\frac{1}{N}$ et $p=2$,

$$
D\left(\frac{H}{N(N+1)(N+2)}\right)=\frac{S(H)}{N(N+1)(N+2)}=\frac{H}{N(N+2)}-\frac{1}{N+1}+\frac{1}{N+2} .
$$

c) Pour $a=\frac{1}{N^{2}}$ et $p=1$,

$$
D\left(\frac{H^{(2)}}{N(N+1)}\right)=\frac{1}{N(N+1)} S\left(\frac{1}{N} H\right)=\frac{H^{2}}{2 N(N+1)}+\frac{H^{(2)}}{2 N(N+1)} .
$$


COROLlaIRe 7. Pour tout réel $p \neq-1,-2, \ldots$, on a

$$
D\left(\frac{1}{(N+p)^{2}}\right)=\frac{p !}{(N)_{p+1}} \bowtie \frac{p !}{(N)_{p+1}}=\frac{p !}{(N)_{p+1}} S\left(\frac{1}{N+p}\right) .
$$

Démonstration. Par (3.9),

$$
\frac{p !}{(N)_{p+1}} \bowtie \frac{p !}{(N)_{p+1}}=\frac{p !}{(N)_{p+1}} S\left(\frac{(N)_{p}}{p !} \frac{p !}{(N)_{p+1}}\right)=\frac{p !}{(N)_{p+1}} S\left(\frac{1}{N+p}\right) .
$$

EXEMPLE 16.

a) Pour $p=1$,

$$
D\left(\frac{1}{(N+1)^{2}}\right)=\frac{1}{N(N+1)} S\left(\frac{1}{N+1}\right)=\frac{H}{N(N+1)}-\frac{1}{(N+1)^{2}},
$$

ce qui peut se réécrire:

$$
\frac{H}{N(N+1)}=\frac{1}{(N+1)^{2}}+D\left(\frac{1}{(N+1)^{2}}\right) \text {. }
$$

b) Pour $p=-\frac{1}{2}$,

$$
D\left(\frac{1}{\left(N-\frac{1}{2}\right)^{2}}\right)=\frac{\Gamma\left(\frac{1}{2}\right)}{(N)_{\frac{1}{2}}} S\left(\frac{1}{N-\frac{1}{2}}\right)
$$

ce qui peut se réécrire:

$$
D\left(\frac{1}{(2 N-1)^{2}}\right)=\frac{2^{2 N-1}}{N\left(\begin{array}{c}
2 N \\
N
\end{array}\right)} O
$$

c'est-à-dire

$$
D\left(\frac{1}{(2 N-1)^{2}}\right)(n)=\frac{2^{2 n-1}}{n\left(\begin{array}{c}
2 n \\
n
\end{array}\right)} \sum_{k=1}^{n} \frac{1}{2 k-1} \quad \text { (cf. [1], p.293 (34.3)) }
$$

REMARQUE 7. Plus généralement, on peut montrer que, pour tout entier $k \geq 1$, on a

$$
D\left(\frac{1}{(N+p)^{k+1}}\right)=\frac{N !}{N(p+1)_{N}} P_{k}\left(S_{p}^{(1)}, \ldots, S_{p}^{(k)}\right),
$$

avec, pour $1 \leq m \leq k$,

$$
S_{p}^{(m)}(n)=\sum_{j=1}^{n} \frac{1}{(p+j)^{m}}
$$


où les $P_{k}\left(X_{1}, \ldots, X_{k}\right)$ sont les polynômes de Bell modifiés (cf. [3], [5]) définis par la fonction génératrice

$$
\exp \left(\sum_{m \geq 1} X_{m} \frac{x^{m}}{m}\right)=\sum_{k \geq 0} P_{k}\left(X_{1}, \ldots, X_{k}\right) x^{k} .
$$

En particulier, pour $p=0$,

$$
D\left(\frac{1}{N^{k+1}}\right)=\frac{1}{N} P_{k}\left(H, H^{(2)}, \ldots, H^{(k)}\right),
$$

et pour $p=-\frac{1}{2}$,

$$
D\left(\frac{1}{(2 N-1)^{k+1}}\right)=\frac{2^{2 N-1}}{N\left(\begin{array}{c}
2 N \\
N
\end{array}\right)} P_{k}\left(O, O^{(2)}, \ldots, O^{(k)}\right) .
$$

\section{LES SOMMES HARMONIQUES}

On rappelle la propriété d'harmonicité (3.8): $\frac{1}{N} \bowtie a=\frac{1}{N} S(a)$. Cette propriété justifie la généralisation suivante.

DÉFINITION 9. Soit une suite $a \in \mathcal{E}^{*}$, on définit pour tout entier naturel $k$, la somme harmonique $k$-ième de a notée $S^{(k)}(a)$ par la formule

$$
\left(\frac{1}{N}\right)^{\bowtie k} \bowtie a=\frac{1}{N} S^{(k)}(a)
$$

EXEMPLE 17.

$$
D\left(\frac{1}{N^{k+1}}\right)=\left(\frac{1}{N}\right)^{\bowtie(k+1)}=\left(\frac{1}{N}\right)^{\bowtie k} \bowtie \frac{1}{N}=\frac{1}{N} S^{(k)}\left(\frac{1}{N}\right) .
$$

D'où

$$
S^{(k)}\left(\frac{1}{N}\right)=N D\left(\frac{1}{N^{k+1}}\right)
$$

ce qui se traduit par

$$
S^{(k)}\left(\frac{1}{N}\right)(n)=n D\left(\frac{1}{N^{k+1}}\right)(n)=\sum_{m=1}^{n}(-1)^{m-1}\left(\begin{array}{c}
n \\
m
\end{array}\right) \frac{1}{m^{k}} .
$$

Plus généralement, on a l'identité suivante, 
Proposition 11. Pour toute suite $a \in \mathcal{E}^{*}$ et pour $k \geq 1$,

$$
S^{(k)}(a)(n)=\sum_{m=1}^{n}(-1)^{m-1}\left(\begin{array}{l}
n \\
m
\end{array}\right) \frac{1}{m^{k-1}} D(a)(m) .
$$

Démonstration. D'après (4.1) et la définition du produit harmonique,

$$
\frac{1}{N} S^{(k)}(a)=\left(\frac{1}{N}\right)^{\bowtie k} \bowtie a=D\left(\frac{1}{N^{k}} D(a)\right)
$$

d'où

ce qui se traduit par

$$
S^{(k)}(a)=N D\left(\frac{1}{N^{k}} D(a)\right)
$$

$$
S^{(k)}(a)(n)=n D\left(\frac{1}{N^{k}} D(a)\right)(n)=\sum_{m=1}^{n}(-1)^{m-1}\left(\begin{array}{l}
n \\
m
\end{array}\right) \frac{1}{m^{k-1}} D(a)(m) .
$$

On va à présent donner une autre expression des sommes harmoniques.

PROPOSITION 12. Pour toute suite $a \in \mathcal{E}^{*}$, on a $S^{(0)}(a)(n)=n a(n)$ et la relation de récurrence:

$$
S^{(k+1)}(a)(n)=\sum_{m=1}^{n} \frac{1}{m} S^{(k)}(a)(m) \quad \text { pour } k \geq 0 .
$$

Il en résulte que $S^{(1)}(a)=S(a)$, et pour $k \geq 1$,

$$
S^{(k)}(a)(n)=\sum_{n \geq n_{1} \geq \cdots \geq n_{k} \geq 1} \frac{1}{n_{1} \ldots n_{k-1}} a\left(n_{k}\right) .
$$

Démonstration. On a $\delta_{0} \bowtie a=\frac{1}{N} S^{(0)}(a)$, c'est-à-dire $S^{(0)}(a)=N a$. Pour $k \geq 0$, on peut écrire par (3.8) et (4.1),

$$
\frac{1}{N} S^{(k+1)}(a):=\left(\frac{1}{N}\right)^{\bowtie(k+1)} \bowtie a=\frac{1}{N} \bowtie\left(\left(\frac{1}{N}\right)^{\bowtie k} \bowtie a\right)=\frac{1}{N} S\left(\left(\frac{1}{N}\right)^{\bowtie k} \bowtie a\right) .
$$

On en déduit la relation de récurrence

$$
S^{(k+1)}(a)=S\left(\left(\frac{1}{N}\right)^{\bowtie k} \bowtie a\right)=S\left(\frac{1}{N} S^{(k)}(a)\right)
$$

qui se traduit par (4.4). La formule (4.5) s'en d éduit aussitôt par récurrence. 
EXEMPLE 18.

$S^{(0)}\left(\frac{1}{N}\right)=\mathbf{1}, \quad S^{(1)}\left(\frac{1}{N}\right)=H, \quad S^{(2)}\left(\frac{1}{N}\right)=S\left(\frac{1}{N} H\right)=\frac{1}{2}\left(H^{2}+H^{(2)}\right)$.

THÉORÈME 4. Pour toute suite $a \in \mathcal{E}^{*}$, et pour $k \geq 1$, on a l'identité

$$
\sum_{n \geq n_{1} \geq \cdots \geq n_{k} \geq 1} \frac{1}{n_{1} \ldots n_{k-1}} a\left(n_{k}\right)=\sum_{m=1}^{n}(-1)^{m-1}\left(\begin{array}{c}
n \\
m
\end{array}\right) \frac{1}{m^{k-1}} D(a)(m) .
$$

Démonstration. La formule (4.6) résulte des formules (4.3) et (4.5).

Corollaire 8 (Formule de Dilcher généralisée). Pour $k \geq 1$ et $q \geq 1$,

$$
\sum_{n \geq n_{1} \geq \cdots \geq n_{k} \geq 1} \frac{1}{n_{1} \ldots n_{k-1}} \frac{1}{n_{k}^{q}}=\sum_{m=1}^{n}(-1)^{m-1}\left(\begin{array}{c}
n \\
m
\end{array}\right) \frac{1}{m^{k}} \sum_{m \geq m_{1} \geq \cdots \geq m_{q-1} \geq 1} \frac{1}{m_{1} \ldots m_{q-1}} .
$$

Démonstration. On applique (4.6) à la suite $a=\frac{1}{N^{q}}$. Par (4.2), on a $D(a)=\frac{1}{N} S^{(q-1)}\left(\frac{1}{N}\right)$.

EXEMPLE 19.

a) $a=\frac{1}{N^{2}}$,

$$
\sum_{n \geq n_{1} \geq \cdots \geq n_{k} \geq 1} \frac{1}{n_{1} \ldots n_{k-1}} \frac{1}{n_{k}^{2}}=\sum_{m=1}^{n}(-1)^{m-1}\left(\begin{array}{c}
n \\
m
\end{array}\right) \frac{H(m)}{m^{k}},
$$

b) $a=\frac{1}{N^{3}}$,

$$
\sum_{n \geq n_{1} \geq \cdots \geq n_{k} \geq 1} \frac{1}{n_{1} \ldots n_{k-1}} \frac{1}{n_{k}^{3}}=\sum_{m=1}^{n}(-1)^{m-1}\left(\begin{array}{c}
n \\
m
\end{array}\right) \frac{(H(m))^{2}+H^{(2)}(m)}{2 m^{k}}
$$

c) $a=\frac{1}{2 N-1}$,

$$
\sum_{n \geq n_{1} \geq \cdots \geq n_{k} \geq 1} \frac{1}{n_{1} \ldots n_{k-1}} \frac{1}{2 n_{k}-1}=\sum_{m=1}^{n}(-1)^{m-1}\left(\begin{array}{c}
n \\
m
\end{array}\right) \frac{2^{2 m-1}}{m^{k}\left(\begin{array}{c}
2 m \\
m
\end{array}\right)},
$$

d) $a=\frac{1}{(2 N-1)^{2}}$,

$$
\sum_{n \geq n_{1} \geq \cdots \geq n_{k} \geq 1} \frac{1}{n_{1} \ldots n_{k-1}} \frac{1}{\left(2 n_{k}-1\right)^{2}}=\sum_{m=1}^{n}(-1)^{m-1}\left(\begin{array}{c}
n \\
m
\end{array}\right) \frac{2^{2 m-1} O(m)}{m^{k}\left(\begin{array}{c}
2 m \\
m
\end{array}\right)} .
$$




\section{LA TRANSFORMATION D'EULER}

\subsection{Transformation D'EULER FORMELLE DANS C $[[z]]$}

THÉORÈME 5. Soit $a \in \mathcal{E}^{*}$, on a la relation dans $\mathbf{C}[[z]]$

$$
\sum_{n \geq 1} D(a)(n) z^{n}=-\sum_{n \geq 1} a(n)\left(\frac{z}{z-1}\right)^{n}
$$

Démonstration. Par définition de $D(a)$, on a

$$
\sum_{n \geq 0} D(a)(n+1) z^{n}=\sum_{n \geq 0} z^{n} \sum_{k=0}^{n}(-1)^{k}\left(\begin{array}{l}
n \\
k
\end{array}\right) a(k+1) .
$$

Or

$$
\begin{aligned}
\sum_{n \geq 0} z^{n} \sum_{k=0}^{n}(-1)^{k}\left(\begin{array}{l}
n \\
k
\end{array}\right) a(k+1) & =\sum_{k \geq 0}(-1)^{k} a(k+1) z^{k} \sum_{n \geq k}\left(\begin{array}{l}
n \\
k
\end{array}\right) z^{n-k} \\
& =\sum_{k \geq 0}(-1)^{k} a(k+1) z^{k}(1-z)^{-k-1} \\
& =\frac{1}{1-z} \sum_{k \geq 0}(-1)^{k} a(k+1)\left(\frac{z}{1-z}\right)^{k} \\
& =\frac{1}{1-z} \sum_{k \geq 0} a(k+1)\left(\frac{z}{z-1}\right)^{k} .
\end{aligned}
$$

D'où

$$
\sum_{n \geq 0} D(a)(n+1) z^{n+1}=\frac{z}{1-z} \sum_{k \geq 0} a(k+1)\left(\frac{z}{z-1}\right)^{k}
$$

qui est la relation cherchée.

EXEMPLE 20. D'après les exemples $15 \mathrm{a}$ ),b),c) et $16 \mathrm{a}$ ), on a les relations suivantes :

a) $\sum_{n \geq 1} \frac{H(n)}{n(n+1)} z^{n}=-\sum_{n \geq 1} \frac{H(n)}{n(n+1)}\left(\frac{z}{z-1}\right)^{n}$

$$
=\sum_{n \geq 1} \frac{1}{(n+1)^{2}} z^{n}-\sum_{n \geq 1} \frac{1}{(n+1)^{2}}\left(\frac{z}{z-1}\right)^{n}
$$

b) $\sum_{n \geq 1} \frac{H(n)}{n(n+2)} z^{n}-\sum_{n \geq 1} \frac{z^{n}}{(n+1)(n+2)}=-\sum_{n \geq 1} \frac{H(n)}{n(n+1)(n+2)}\left(\frac{z}{z-1}\right)^{n}$, 
c) $\frac{1}{2} \sum_{n \geq 1} \frac{(H(n))^{2}}{n(n+1)} z^{n}+\frac{1}{2} \sum_{n \geq 1} \frac{H^{(2)}(n)}{n(n+1)} z^{n}=-\sum_{n \geq 1} \frac{H^{(2)}(n)}{n(n+1)}\left(\frac{z}{z-1}\right)^{n}$.

COROLlaIRE 9. Pour toute suite $a \in \mathcal{E}^{*}$ et tout entier naturel $k$, on a l'identité

$$
\sum_{n \geq 1} \frac{D(a)(n)}{n^{k}} z^{n}=-\sum_{n \geq 1} \frac{1}{n} S^{(k)}(a)(n)\left(\frac{z}{z-1}\right)^{n}
$$

Démonstration. D'après (4.1), on a $\frac{1}{N^{k}} D(a)=D\left(\frac{1}{N} S^{(k)}(a)\right)$. La formule (5.2) résulte alors de (5.1).

EXEMPLE 21.

a) En appliquant (5.2) avec $a=\frac{1}{N}$, on obtient pour $k \geq 1$,

$$
\operatorname{Li}_{k+1}(z)=-\sum_{n \geq 1} \frac{1}{n} \sum_{n \geq n_{1} \geq \cdots \geq n_{k} \geq 1} \frac{1}{n_{1} \ldots n_{k}}\left(\frac{z}{z-1}\right)^{n}
$$

où $\mathrm{Li}_{k}$ désigne (formellement) le polylogarithme

$$
\operatorname{Li}_{k}(z):=\sum_{n \geq 1} \frac{1}{n^{k}} z^{n}
$$

b) En appliquant (5.2) avec $a=\frac{1}{N^{2}}$, on obtient pour $k \geq 1$,

$$
\text { (5.4) } \sum_{n \geq 1} \frac{H(n)}{n^{k+1}} z^{n}=-\sum_{n \geq 1} \frac{1}{n} \sum_{n \geq n_{1} \geq \cdots \geq n_{k} \geq 1} \frac{1}{n_{1} \ldots n_{k-1}} \frac{1}{n_{k}^{2}}\left(\frac{z}{z-1}\right)^{n} \text {. }
$$

c) En appliquant (5.2) avec $a=\frac{1}{2 N-1}$, on obtient pour $k \geq 1$,

$$
\text { (5.5) } \begin{aligned}
\frac{1}{2} \sum_{n \geq 1} \frac{2^{2 n}}{\left(\begin{array}{c}
2 n \\
n
\end{array}\right)} & \frac{1}{n^{k+1}} z^{n} \\
& =-\sum_{n \geq 1} \frac{1}{n} \sum_{n \geq n_{1} \geq \cdots \geq n_{k} \geq 1} \frac{1}{n_{1} \ldots n_{k-1}} \frac{1}{2 n_{k}-1}\left(\frac{z}{z-1}\right)^{n} .
\end{aligned}
$$


d) En appliquant (5.2) avec $a=\frac{1}{(2 N-1)^{2}}$, on obtient pour $k \geq 1$,

$$
\text { (5.6) } \begin{aligned}
\frac{1}{2} \sum_{n \geq 1} \frac{2^{2 n}}{\left(\begin{array}{c}
2 n \\
n
\end{array}\right)} \frac{O(n)}{n^{k+1}} z^{n} \\
\quad=-\sum_{n \geq 1} \frac{1}{n} \sum_{n \geq n_{1} \geq \cdots \geq n_{k} \geq 1} \frac{1}{n_{1} \ldots n_{k-1}} \frac{1}{\left(2 n_{k}-1\right)^{2}}\left(\frac{z}{z-1}\right)^{n} .
\end{aligned}
$$

\subsection{TRANSFORMATION D'EULER ANALYTIQUE}

THÉORÈME 6. Soit une suite $a \in \mathcal{E}^{*}$. Si la série $\sum_{n \geq 1} a(n) z^{n}$ est convergente dans le disque unité $\mathbf{D}(0,1)$ alors la série $\sum_{n \geq 1} \bar{D}(a)(n) z^{n}$ est convergente dans le disque ouvert $\mathbf{D}\left(0, \frac{1}{2}\right)$ et on a pour tout $z \in \mathbf{D}\left(0, \frac{1}{2}\right)$

$$
\sum_{n=1}^{+\infty} D(a)(n) z^{n}=-\sum_{n=1}^{+\infty} a(n)\left(\frac{z}{z-1}\right)^{n}
$$

L'application $z \mapsto \frac{z}{z-1}$ étant involutive, il en résulte que si la série $\sum_{n \geq 1} D(a)(n) z^{n}$ est convergente dans le disque $\mathbf{D}(0,1)$ alors la série $\sum_{n \geq 1} a(n) z^{n}$ est convergente dans le disque ouvert $\mathbf{D}\left(0, \frac{1}{2}\right)$ et on a pour tout $z \in \mathbf{D}\left(0, \frac{1}{2}\right)$

$$
\sum_{n=1}^{+\infty} a(n) z^{n}=-\sum_{n=1}^{+\infty} D(a)(n)\left(\frac{z}{z-1}\right)^{n}
$$

Démonstration. Pour $z \in \mathbf{D}(0,1)$, posons $A(z)=\sum_{n=0}^{+\infty} a(n+1) z^{n}$. On a pour tout $0<r<1$ et pour tout entier $k \geq 0$

$$
a(k+1)=\frac{1}{2 i \pi} \int_{\mathbf{C}(0, r)} \frac{A(u)}{u^{k+1}} d u,
$$

où $\mathbf{C}(0, r)$ est le cercle paramétré par $t \mapsto r e^{i t}$, avec $t \in[0,2 \pi]$. On en déduit que pour tout entier $n \geq 1$ on a

$$
D(a)(n+1)=\frac{1}{2 i \pi} \int_{\mathbf{C}(0, r)}\left(1-\frac{1}{u}\right)^{n} \frac{A(u)}{u} d u .
$$

On va montrer que

$$
\sum_{n \geq 0} D(a)(n+1) z^{n+1}=\frac{1}{2 i \pi} \int_{\mathbf{C}(0, r)} \sum_{n \geq 0}\left(z\left(1-\frac{1}{u}\right)\right)^{n} A(u) \frac{z}{u} d u
$$


Pour cela, il suffit que la série $\sum_{n \geq 0}\left(z\left(1-\frac{1}{u}\right)\right)^{n} A(u) \frac{z}{u}$ soit normalement convergente sur le cercle $\mathbf{C}(0, r)$, ce qui est le cas si

$$
|z|<\left|\frac{u}{u-1}\right|
$$

Or, si $u \in \mathbf{C}(0, r)$, on a $\frac{u}{u-1} \in \mathbf{C}\left(\frac{r^{2}}{r^{2}-1}, \frac{r}{1-r^{2}}\right)$, donc $\left|\frac{u}{u-1}\right| \geq \frac{r}{r+1}$.

On en déduit que si $|z|<\frac{r}{r+1}$, alors on a

$$
\begin{aligned}
\sum_{n \geq 0} D(a)(n+1) z^{n+1} & =\frac{1}{2 i \pi} \int_{\mathbf{C}(0, r)} \sum_{n \geq 0}\left(z\left(1-\frac{1}{u}\right)\right)^{n} A(u) \frac{z}{u} d u \\
& =\frac{-1}{2 i \pi} \int_{\mathbf{C}(0, r)} \frac{z}{u z-z-u} A(u) d u .
\end{aligned}
$$

Comme $0<r<1$, ceci prouve que la série $\sum_{n \geq 0} D(a)(n+1) z^{n+1}$ est convergente dans le disque $\mathbf{D}\left(0, \frac{1}{2}\right)$. D'autre part,

$$
\frac{z}{u z-z-u} A(u)=\frac{z}{z-1} \frac{1}{1-\frac{1}{u} \frac{z}{z-1}} \frac{A(u)}{u}=\sum_{n \geq 0}\left(\frac{z}{z-1}\right)^{n+1} \frac{A(u)}{u^{n+1}} .
$$

Cette dernière série converge normalement sur $\mathbf{C}(0, r)$ si

$$
\left|\frac{z}{z-1}\right|<|u|=r
$$

ce qui est le cas si $z \in \mathbf{D}\left(\frac{r^{2}}{r^{2}-1}, \frac{r}{1-r^{2}}\right)$.

En conclusion, si $z \in \mathbf{D}\left(\frac{r^{2}}{r^{2}-1}, \frac{r}{1-r^{2}}\right) \cap \mathbf{D}\left(0, \frac{r}{r+1}\right)=\mathbf{D}\left(0, \frac{r}{r+1}\right)$, alors on a

$$
\begin{aligned}
\sum_{n \geq 0} D(a)(n+1) z^{n+1} & =\sum_{n \geq 0}\left(\frac{z}{z-1}\right)^{n+1} \frac{1}{2 i \pi} \int_{\mathbf{C}(0, r)} \frac{A(u)}{u^{n+1}} d u \\
& =-\sum_{n \geq 0}\left(\frac{z}{z-1}\right)^{n+1} a(n+1)
\end{aligned}
$$

Comme $0<r<1$, ceci prouve qu'on a l'égalité dans le disque $\mathbf{D}\left(0, \frac{1}{2}\right)$.

Par le Lemme d'Abel sur les séries entières, on déduit du théorème précédent le corollaire suivant. 
COROLlaire 10.

(1) Si les séries $\sum_{n \geq 1} a(n)(-1)^{n}$ et $\sum_{n \geq 1} D(a)(n)\left(\frac{1}{2}\right)^{n}$ convergent, alors on a l'égalité

$$
\sum_{n=1}^{+\infty} D(a)(n)\left(\frac{1}{2}\right)^{n}=\sum_{n=1}^{+\infty}(-1)^{n-1} a(n)
$$

(2) Si les séries $\sum_{n \geq 1} D(a)(n)(-1)^{n}$ et $\sum_{n \geq 1} a(n)\left(\frac{1}{2}\right)^{n}$ convergent, alors on a l'égalité

$$
\sum_{n=1}^{+\infty}(-1)^{n-1} D(a)(n)=\sum_{n=1}^{+\infty} a(n)\left(\frac{1}{2}\right)^{n}
$$

EXEMPLE 22. D'après l'exemple $20 \mathrm{a}$ ), on a (cf. [1], p. 248)

$$
\sum_{n=1}^{\infty} \frac{H(n)}{2^{n} n(n+1)}=\sum_{n=1}^{\infty} \frac{(-1)^{n-1} H(n)}{n(n+1)}=2 \mathrm{Li}_{2}\left(\frac{1}{2}\right)+\mathrm{Li}_{2}(-1)=\frac{1}{2} \zeta(2)-\log ^{2}(2) .
$$

COROllaire 11. Si la série $\sum_{n \geq 1} a(n) z^{n}$ est convergente dans le disque $\mathbf{D}(0,1)$, alors on a pour tout $z \in \mathbf{D}\left(0, \frac{1}{2}\right)$ et pour $k \geq 0$,

$$
\sum_{n=1}^{\infty} \frac{D(a)(n)}{n^{k}} z^{n}=-\sum_{n=1}^{\infty} \frac{1}{n} S^{(k)}(a)(n)\left(\frac{z}{z-1}\right)^{n} .
$$

En particulier, pour $k=1$,

$$
\sum_{n=1}^{\infty} \frac{D(a)(n)}{n} z^{n}=-\sum_{n=1}^{\infty} \frac{1}{n} S(a)(n)\left(\frac{z}{z-1}\right)^{n} .
$$

De plus, si les séries $\sum_{n=1}^{\infty} \frac{D(a)(n)}{n^{k}} \frac{1}{2^{n}}$ et $\sum_{n=1}^{\infty} \frac{1}{n} S^{(k)}(a)(n)(-1)^{n}$ convergent, alors on a l'égalité

$$
\sum_{n=1}^{\infty} \frac{(-1)^{n-1}}{n} S^{(k)}(a)(n)=\sum_{n=1}^{\infty} \frac{D(a)(n)}{2^{n} n^{k}} .
$$

Démonstration. Si la série $\sum_{n \geq 1} a(n) z^{n}$ converge dans le disque $\mathbf{D}(0,1)$, alors il en est de même de la série $\sum_{n \geq 1} \frac{1}{n} S^{(k)}(a)(n) z^{n}$. Cela résulte de la relation de récurrence (cf. (4.4)) :

$$
\frac{1}{N} S^{(k+1)}(a)=\frac{1}{N} S\left(\frac{1}{N} S^{(k)}(a)\right),
$$


et du fait que si une série $\sum_{n \geq 1} b(n) z^{n}$ converge dans le disque $\mathbf{D}(0,1)$, alors il en est de même de la série $\sum_{n \geq 1} \frac{1}{n} S(b)(n) z^{n}$. On peut alors appliquer le Théorème 6 car $D\left(\frac{1}{N} S^{(k)}(a)\right)=\frac{1}{N^{k}} D(a)$.

EXEMPLE 23.

a) Pour $a=\frac{1}{N}$, on a $D(a)=a$, d'où pour $k=1$,

$$
\sum_{n=1}^{\infty}(-1)^{n-1} \frac{H(n)}{n}=\operatorname{Li}_{2}\left(\frac{1}{2}\right)=\frac{1}{2} \zeta(2)-\frac{1}{2} \log ^{2}(2) \quad \text { (cf. [1], p. 248), }
$$

et pour $k=2$, (cf. [1],p. 249)

$$
\sum_{n=1}^{\infty} \frac{(-1)^{n-1}}{n} \sum_{m=1}^{n} \frac{H(m)}{m}=\mathrm{Li}_{3}\left(\frac{1}{2}\right)=\frac{1}{6}(\log 2)^{3}-\frac{1}{2} \zeta(2) \log 2+\frac{7}{8} \zeta(3) .
$$

b) Pour $a=\frac{1}{N^{2}}$, on a $D(a)=\frac{1}{N} H$, d'où pour $k=0$,

$$
\sum_{n=1}^{\infty}(-1)^{n-1} \frac{1}{n^{2}}=\sum_{n=1}^{\infty} \frac{H(n)}{2^{n} n}=\frac{1}{2} \zeta(2)
$$

pour $k=1$,

$$
\sum_{n=1}^{\infty}(-1)^{n-1} \frac{H^{(2)}(n)}{n}=\sum_{n=1}^{\infty} \frac{H(n)}{2^{n} n^{2}}=\zeta(3)-\frac{1}{2} \zeta(2) \log 2 \quad \text { (cf. [1], p.258), }
$$

et pour $k=2$,

$$
\sum_{n=1}^{\infty} \frac{(-1)^{n-1}}{n} \sum_{m=1}^{n} \frac{H^{(2)}(m)}{m}=\sum_{n=1}^{\infty} \frac{H(n)}{2^{n} n^{3}} .
$$

c) Pour $a=\frac{1}{2 N-1}$, on a $D(a)=\frac{1}{N} \frac{2^{2 N-1}}{\left(\begin{array}{c}2 N \\ N\end{array}\right)}$, d'où pour $k=0$,

$$
\sum_{n=1}^{\infty} \frac{(-1)^{n-1}}{2 n-1}=\frac{1}{2} \sum_{n=1}^{\infty} \frac{2^{n}}{n\left(\begin{array}{c}
2 n \\
n
\end{array}\right)}=\frac{\pi}{4} \quad \text { (formule d'Euler: cf. [8]), }
$$

pour $k=1$, (formule de Jean Bernoulli : cf. [8])

$$
\sum_{n=1}^{\infty}(-1)^{n-1} \frac{O(n)}{n}=\frac{1}{2} \sum_{n=1}^{\infty} \frac{2^{n}}{n^{2}\left(\begin{array}{c}
2 n \\
n
\end{array}\right)}=\frac{\pi^{2}}{16}
$$


pour $k=2$, (cf. [4] (2.67))

$$
\sum_{n=1}^{\infty} \frac{(-1)^{n-1}}{n} \sum_{m=1}^{n} \frac{O(m)}{m}=\frac{1}{2} \sum_{n=1}^{\infty} \frac{2^{n}}{n^{3}\left(\begin{array}{c}
2 n \\
n
\end{array}\right)}=\frac{\pi^{2}}{16} \log 2+\frac{\pi G}{2}-\frac{35}{32} \zeta(3),
$$

où $G$ désigne la constante de Catalan :

$$
G=\sum_{n=1}^{\infty} \frac{(-1)^{n-1}}{(2 n-1)^{2}}
$$

d) Pour $a=\frac{1}{(2 N-1)^{2}}$, on a $D(a)=\frac{1}{N} \frac{2^{2 N-1}}{\left(\begin{array}{c}2 N \\ N\end{array}\right)} O$, d'où pour $k=0$,

$$
\frac{1}{2} \sum_{n=1}^{\infty} \frac{2^{n}}{\left(\begin{array}{c}
2 n \\
n
\end{array}\right)} \frac{O(n)}{n}=G
$$

(formule de Ramanujan pour la constante de Catalan : cf. [1], p.293-294). Pour $k=1$, (cf. [4] (2.36) et (2.37))

$$
\sum_{n=1}^{\infty} \frac{(-1)^{n-1}}{n} O^{(2)}(n)=\frac{1}{2} \sum_{n=1}^{\infty} \frac{2^{n}}{\left(\begin{array}{c}
2 n \\
n
\end{array}\right)} \frac{O(n)}{n^{2}}=\frac{7}{4} \zeta(3)-\frac{\pi G}{2},
$$

pour $k=2$,

$$
\sum_{n=1}^{\infty} \frac{(-1)^{n-1}}{n} \sum_{m=1}^{n} \frac{O^{(2)}(m)}{m}=\frac{1}{2} \sum_{n=1}^{\infty} \frac{2^{n}}{\left(\begin{array}{c}
2 n \\
n
\end{array}\right)} \frac{O(n)}{n^{3}} .
$$

\section{BIBLIOGRAPHIE}

[1] Berndt, B.C. Ramanujan's Notebooks. Part I. Springer-Verlag, New York, 1985.

[2] BOYADZHIEV, K. N. Harmonic number identities via Euler's transform. J. Integer Seq. 12 (2009), Article 09.6.1, 8 pp.

[3] CANDElPERgher, B. and M.-A. Coppo. A new class of identities involving Cauchy numbers, harmonic numbers and zeta values. Ramanujan J. 27 (2012), 305-328.

[4] Davydychev, A.I. and M.Yu. Kalmykov. Massive Feynman diagrams and inverse binomial sums. Nuclear Phys. B 699 (2004), 3-64.

[5] Flajolet, P. and R. SedGewick. Mellin transforms and asymptotics: finite differences and Rice's integrals. Theoret. Comput. Sci. 144 (1995), 101124.

[6] Hardy, G.H. Divergent Series. Oxford, Clarendon Press, 1963. (First edition 1949 , third, corrected edition published in 1963.) 
[7] Henrici, P. Applied and Computational Complex Analysis. Volume 1: Power series-integration-conformal mapping-location of zeros. Pure and Applied Mathematics. Wiley-Interscience, New York-London-Sydney, 1974.

[8] LEHMER, D.H. Interesting series involving the central binomial coefficient. Amer. Math. Monthly 92 (1985), 449-457.

[9] LoEB, D.E. and G.-C. RotA. Formal power series of logarithmic type. $A d v$. Math. 75 (1989), 1-118.

[10] Roman, S. The logarithmic binomial formula. Amer. Math. Monthly 99 (1992), 641-648.

[11] SuN, Z.-H. Invariant sequences under binomial transformation. Fibonacci Quart. 39 (2001), 324-333.

(Reçu le 29 juin 2011)

Bernard Candelpergher et Marc-Antoine Coppo

Université de Nice-Sophia Antipolis

Laboratoire Jean-Alexandre Dieudonné

Parc Valrose

F-06108 Nice Cedex 2

France

e-mail: Bernard.Candelpergher@unice.fr

e-mail: Marc-Antoine.Coppo@unice.fr 